\title{
Nonergodic Dynamics of a Novel Thermally Sensitive Hybrid Gel
}

\author{
Yue Zhao, ${ }^{\dagger}$ Guangzhao Zhang, ${ }^{\ddagger}$ and Chi Wu*,t, \\ The Opening Laboratory for Bond-sel ective Chemistry, Department of Chemical Physics, University of \\ Science and Technology of China, Hefei, Anhui, 230026, China, and Department of Chemistry, \\ The Chinese University of Hong Kong, Shatin, N.T., Hong Kong
}

Received April 17, 2001; Revised Manuscript Received August 24, 2001

\begin{abstract}
As the temperature decreases, the swelling of individual thermally sensitive spherical poly( $\mathrm{N}$-isopropylacrylamide) microgel s in a concentrated dispersion can lead to a sol-gel transition, resulting in a hybrid bulk gel in which individual microgels were physically jam-packed into a three-dimensional network without involving electrostatic interaction, resembling the glass transition of small molecules. The dynamics of such a formed hybrid gel were studied by static and dynamic laser light scattering. Our results showed that the inhomogeneity should be characterized by the difference between the ensemble average scattering intensity and the dynamic component of the time average scattering intensity. The inhomogeneity was strongly dependent on the sol-gel transition rate and the gelling temperature. Our results have also revealed that the inhomogeneity can be completely suppressed if individual swollen microgels are closely packed together, indicating that the static nonergodic behavior originates from large voids formed during the sol-gel transition.
\end{abstract}

\section{Introduction}

A gel is generally considered to be a three-dimensional polymer network swollen by a large amount of solvent. In one way, polymer gels can be generally classified into so-called "chemical" and "physical" gels. A chemical gel is normally formed via the copolymeriziation of monomers with a cross-linking agent, in which polymer chains are interconnected by covalent bonds. The formation of a physical gel usually starts with a uniform polymer solution, in which different chains are bound together via physical interaction, e.g., hydrogen bonding, electrostatic interaction, and coordination. The interaction is often induced by an alternation of physical conditions, such as temperature and ionic strength.

It has been well-known that in the formation of chemical gels short linear chains are first formed, then large branched clusters, and finally a network, in which the clusters ("islands") are interconnected to form a "continent", while the solvent "sea" changed into "lakes". ${ }^{1-4}$ In principle, such an inhomogeneous clustering structure could be avoided if long polymer chains uniformly distributed in a solution are randomly associated to form a physical gel. Recently, dynamic light scattering has been used to study a nonergodic soli d like medium, such as polymer gels or colloidal glasses, ${ }^{5}$ wherein the Brownian motions of scattering elements are limited around fixed average positions. One obvious characteristic of the nonergodicity is the appearance of speckles; namely, the scattered intensity depends on the sample position. It is now generally known that the concentration fluctuation inside a gel comprises both static and dynamic parts. The inhomogeneous static part represents a frozen structure, while the dynamic fluctuation presumably reflects diffusive relaxation. ${ }^{6-13}$ The concept of the nonergodicity, stated by Pusey and Van Megen,14 has led to the development of several relevant methods to analyze dynamic properties of gels. ${ }^{15-17}$

† University of Science and Technology of China.

\# The Chinese University of Hong Kong.

* The Hong Kong address should be used for correspondence.
To have a better understanding of the structure and the sol-gel transition of physical gels, we have developed a novel hydrogel, in which billions of small spherical swollen poly( $\mathrm{N}$-isopropylacrylamide) (PNIPAM) microgels $(\sim 100 \mathrm{~nm})$ in a concentrated dispersion are jampacked into a macroscopic three-dimensional gel network. Inside each microgel, the polymer chains were chemically cross-linked, but the microgels were jam-packed by physical interaction. This is why we named it as a "hybrid" gel. It resembles a glass in which atoms or small molecules are replaced by "large" spherical microgels. There are several advantages of using this hybrid gel as a model system to study the volumeconcentration induced sol-gel transition and structures of physical gels. For example, the structure inhomogeneity resulted in the microgel preparation is limited within a volume $\left(\sim 100 \times 100 \times 100 \mathrm{~nm}^{3}\right)$, which is so small in comparison with the light wavelength that it will not show up in laser light scattering. Also, as building blocks, these microgels are narrowly distributed and well characterized. Moreover, such a gelation is completely thermally reversible without any hysteresis. Using a combination of static and dynamic laser light scattering, we studied a series of such hybrid gels formed at different gelling rates and temperatures. The main finding is that the observed static nonergodicity is not intrinsic but strongly depends on how the microgels (clusters) are packed. Therefore, it can be completely suppressed if there are no large void among the microgels. On the other hand, the dynamic fluctuation is nearly not influenced by the static nonergodicity.

\section{Theoretical Background}

For a nonergodic gel, the time-average scattered intensity $\langle I\rangle_{T}$ for each chosen sample position at a given scattering vector q comprises both static and dynamic contributions, $\langle I\rangle_{\mathrm{s}}$ and $\langle\mathrm{I}\rangle_{\mathrm{d}}$, i.e., ${ }^{14}$

$$
\langle I\rangle_{\mathrm{T}}=\langle\mathrm{I}\rangle_{\mathrm{s}}+\langle\mathrm{I}\rangle_{\mathrm{d}}
$$

where $\langle I\rangle_{d}$ is independent of the sample position, but not $\langle I\rangle_{s}$. Therefore, at a given $\mathrm{q}$, for each chosen sample 
position, we can measure one $\langle 1\rangle_{T}$ and one normalized intensity-intensity time correlation function $S(q, \tau)$ defined as ${ }^{14-16}$

$$
\mathrm{S}(\mathrm{q}, \tau)=\frac{\langle\mathrm{I}(\mathrm{q}, 0) \mathrm{I}(\mathrm{q}, \tau)\rangle}{\langle\mathrm{I}(\mathrm{q}, 0)\rangle^{2}}-1
$$

where $\tau$ is the delay time. $\mathbf{S}(q, \tau)$ can be written as $14,16,18,19$

$$
\begin{aligned}
& S(q, \tau)=\left(\frac{\langle I\rangle_{d}}{\langle I\rangle_{T}}\right)^{2} \exp (\left.-2 D q^{2} \tau\right)+ \\
& 2\left(\frac{\langle I\rangle_{d}}{\langle I\rangle_{T}}\right)\left[1-\left(\frac{\langle I\rangle_{d}}{\langle I\rangle_{T}}\right)\right] \exp \left(-D q^{2} \tau\right)
\end{aligned}
$$

where $\mathrm{D}$ is the collective diffusion coefficient. Therefore, for a nonergodic system at a given $q$, one can measure the time-average scattered intensity from different sample positions to obtain an ensemble average scattered intensity, $\langle I\rangle_{E}$. Note that for a given $\mathrm{q},\langle\mathrm{I}\rangle_{T}$ depends on the sample position, but not $\langle I\rangle_{E}$ by its definition: $\langle I\rangle_{E}$ $=\sum\langle\mathrm{I}\rangle_{\mathrm{T}, \mathrm{i}} / \mathrm{N}$, where $\mathrm{i}=1,2, \ldots, \mathrm{N}$, and $\mathrm{N}$ is the number of the positions measured. Only in an ergodic system, $\langle I\rangle_{\mathrm{E}}=\langle\mathrm{I}\rangle_{\mathrm{T}}$. In the dynamic measurement, the initial slope of each In S(q, $\tau)$ vs $\tau$ leads to an apparent diffusion coefficient $D_{A}$ in the range of $D / 2<D_{A}<D$. It has been shown that $D$ and $\langle I\rangle_{d}$ are related to $D_{A}$ and $\langle I\rangle_{T}$ as $s^{14,16}$

$$
\frac{\langle I\rangle_{T}}{D_{A}}=\frac{2\langle I\rangle_{T}}{D}-\frac{\langle I\rangle_{d}}{D}
$$

Experimentally, for each chosen sample position at a given $\mathrm{q}$, one can measure one $\langle\mathrm{I}\rangle_{\mathrm{T}}$ from static LLS and calculate one $\mathrm{D}_{\mathrm{A}}$ from $\mathrm{S}(\mathrm{q}, \tau)$ measured in dynamic $\mathrm{LLS}$. Therefore, on the basis of eq $4, D$ and $\langle I\rangle_{d}$ can be respectively obtained from the slope and the intercept of the plot of $\langle I\rangle_{T} / D_{A}$ vs $\langle I\rangle_{T}$ by measuring at a number of sample positions.

\section{Experimental Section}

Sample Preparation. The P NI PAM microgel preparation was developed by Pelton et al. ${ }^{20} \mathrm{~N}$-I sopropylacrylamide was recrystallized three times in a benzene/n-hexane mixture. $\mathrm{N}, \mathrm{N}^{\prime}$-M ethylenebis(acrylamide) (BIS, from Aldrich) as crosslinking agent was recrystallized from methanol. Potassium persulfate (KPS, from Aldrich) as initiator and anionic surfactant sodium dodecyl sulfate (SDS) as dispersant were used without further purification. $4.0 \mathrm{~g}$ of NIPAM, $0.076 \mathrm{~g}$ of BIS, and $0.076 \mathrm{~g}$ of SDS were dissolved into $250 \mathrm{~mL}$ of deionized water in a $500 \mathrm{~mL}$ reactor fitted with a nitrogen bubbling inlet and outl et, a magnet stirrer, and a reflux condenser. After the solution was stirred for $40 \mathrm{~min}$ at $70{ }^{\circ} \mathrm{C}$ under a nitrogen purge, $0.155 \mathrm{~g}$ of KPS dissolved in $20 \mathrm{~mL}$ of deionized water was introduced to start the polymerization. The reaction mixture was kept at $70{ }^{\circ} \mathrm{C}$ for $8 \mathrm{~h}$. The unreacted monomer and most of SDS were removed by adding the reaction mixture dropwise into $250 \mathrm{~mL}$ of methanol. The resulting precipitate was dissolved in $80 \mathrm{~mL}$ of deionized water and was reprecipitated in an equal volume of methanol. To remove the remaining trace amount of SDS and other impurities, the microgels were further purified by three cycles of successive centrifugation, decantation, and redispersion in deionized water. ${ }^{21}$ The resultant dispersion had a $\xi$-potential very close to zero. The purified dispersion was condensed to $49.5 \mathrm{mg} / \mathrm{mL}$.

Laser Light Scattering. A commercial LLS spectrometer (ALV/SP-125) equipped with an ALV-5000 multi- $\tau$ digital time correlator and a solid laser (DPSS, output power $\cong 400 \mathrm{~mW}$ at $\lambda=532 \mathrm{~nm}$ ) was used. In static LLS, the measurement time for each given scattering angle and sample position was $30 \mathrm{~s}$. In dynamic LLS, the high coherence optics was used and the

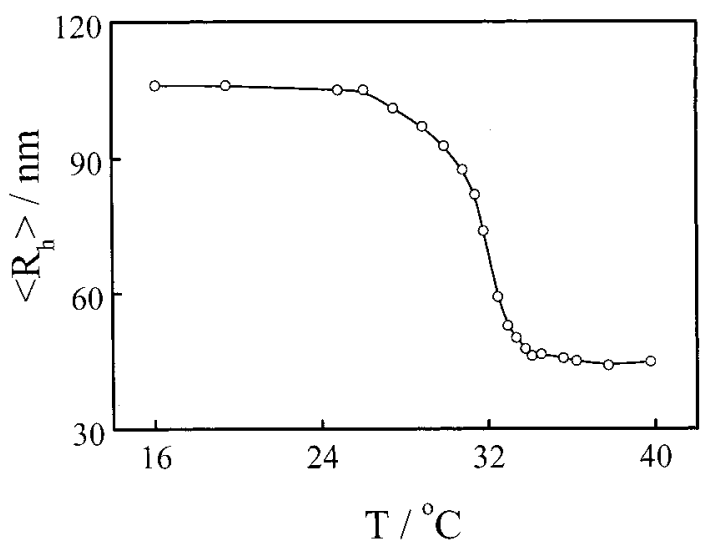

Figure 1. Temperature dependence of average hydrodynamic radius $\left\langle R_{h}\right\rangle$ of microgels in a dilute dispersion, where the microgel concentration is $6.06 \times 10^{-5} \mathrm{~g} / \mathrm{mL}$.

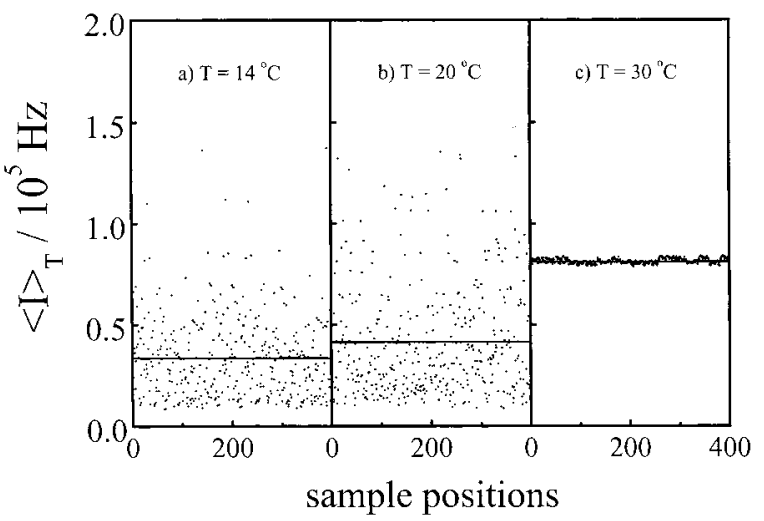

Figure 2. Sample position dependence of time-average scattered light intensity $\langle I\rangle_{T}$ of hybrid PNIPAM gel after the microgel dispersion was quickly cooled from $40{ }^{\circ} \mathrm{C}$ to different gelling temperatures, where the scattering angle $(\theta)$ is $90^{\circ}$, and each solid line represents an ensemble-average scattered light intensity $\langle\mathrm{I}\rangle_{\mathrm{E}}$ defined as $\left(\sum_{\mathrm{i}}\langle\mathrm{I}\rangle_{T, \mathrm{i}}\right) / \mathrm{N}$ with $\mathrm{N}$ being the total number of randomly chosen sample positions.

measurement time was in the range 30-60 min. The Laplace inversion of a measured intensity-intensity time correlation function $\mathrm{G}^{(2)}(\mathrm{t}, \mathrm{q})$ in the self-beating mode can result in a linewidth distribution $\mathrm{G}(\Gamma){ }^{21,22} \mathrm{~F}$ or a pure diffusive relaxation, $\Gamma$ is related to the translational diffusion coefficient $D$ by $\Gamma / q^{2}=$ $\mathrm{D}$ at $\mathrm{q} \rightarrow 0$ and $\mathrm{C} \rightarrow 0$ or a hydrodynamic radius $\mathrm{R}_{\mathrm{h}}$ by the Stokes-Einstein equation: $\mathrm{R}_{\mathrm{h}}=\mathrm{k}_{\mathrm{B}} \mathrm{T} /(6 \pi \eta \mathrm{D})$. The details of LLS theory and instrumentation can be found el sewhere. ${ }^{22-24}$

\section{Results and Discussion}

Figure 1 shows the temperature dependence of the average hydrodynamic radius of the microgels in a dilute solution $\left(6.06 \times 10^{-5} \mathrm{~g} / \mathrm{mL}\right)$. In the temperature range studied, the swelling and shrinking are reversible. The size change between $\sim 45$ and $\sim 106 \mathrm{~nm}$ corresponds to a 13-time change in volume. In such a dilute dispersion, the microgels only took up $0.02-0.26 \%$ volume of the dispersion, so that there is nearly no interparticle interaction. $\Gamma / q^{2}$ was independent of the scattering angle. The ratio of the average radius of gyration $\left\langle R_{g}\right\rangle$ to the average hydrodynamic radius $\left\langle R_{h}\right\rangle$ is close to 0.8 , indicating that the microgels are spherical-like. When the dispersion is concentrated to $4.95 \times 10^{-2} \mathrm{~g} / \mathrm{mL}$, the swelling of individual microgels at lower temperatures $\left(<\sim 25{ }^{\circ} \mathrm{C}\right)$ could lead to a volume-concentration induced sol-gel transition, in which billions of swollen microgels are jam-packed to a macroscopic bulk gel.

Figure 2 shows that in the gel state $(T=14$ and 20 $\left.{ }^{\circ} \mathrm{C}\right)$ the time-average scattered intensity $\langle\mathrm{I}\rangle_{\mathrm{T}}$ randomly 


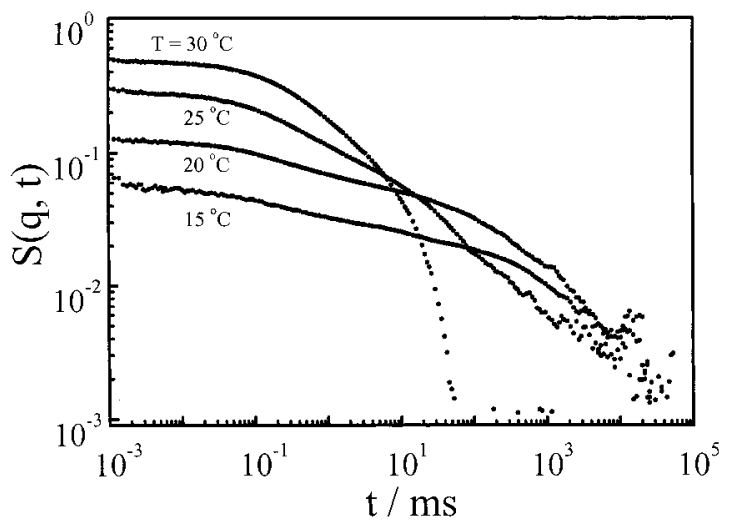

Figure 3. Normalized intensity-intensity time correlation functions $S(q, \tau)$ of the hybrid gels formed at different gelling temperatures, where $\theta=90^{\circ}$.

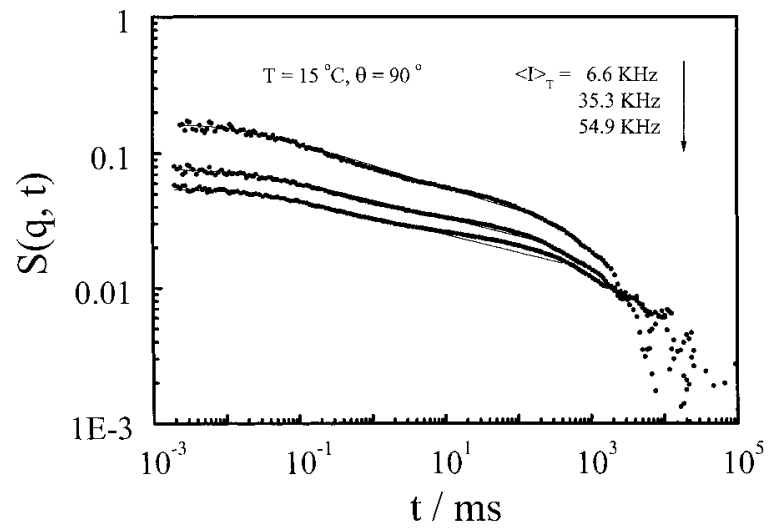

Figure 4. Sample position dependence of normalized intensity-intensity time correlation functions $S(q, \tau)$ of the hybrid gel formed at $15^{\circ} \mathrm{C}$, where $\theta=90^{\circ}$.

varies with the sample position, where the sample position was arbitrarily chosen by rotating and lifting the sample cuvette. While at $\mathrm{T}=30{ }^{\circ} \mathrm{C}$, the speckle pattern disappears because the system becomes a uniform microgel dispersion (the sol state), in which the volume fraction of collapsed microgels is $\sim 16.2 \%$ due to the shrinking of individual microgels. Note that $\langle I\rangle_{E}$ increases as the hybrid gel melts into a dispersion. This is very different from previous observations in which $\langle I\rangle_{E}$ normally decreased as a physical gel melted into individual less scattered polymer chains. ${ }^{4}$ This difference can be attributed to the fact that in this study individual collapsed microgels are much stronger scattering objects than swollen polymer chains in the gel network.

Figure 3 shows that the time correlation function at $30{ }^{\circ} \mathrm{C}$ has only a single expected relaxation with a characteristic time $(\tau)$ of $\sim 1 \mathrm{~ms}$ because individual mi crogels are under Brownian motion in the sol. When the hybrid gel was formed at $\mathrm{T}<25^{\circ} \mathrm{C}$, the second relaxation appeared at $\sim 100 \mathrm{~ms}$, which is $\sim 100$ times slower. The first relaxation might be related to the relaxation of individual microgels inside the hybrid gel network because it has a similar time scale as the microgels in dilute dispersion, while the second relaxation could be attributed to the fluctuation of the jampacked gel network. This is why the second relaxation becomes slower as the gelling temperature decreases. Figure 4 shows that after the gelation the time correlation function depends on the sample position due to its inhomogeneity or nonergodic nature. The plot of " $\mathrm{S}(\mathrm{q}, \mathrm{t})$ vs $t$ " has a power-law behavior over several orders of

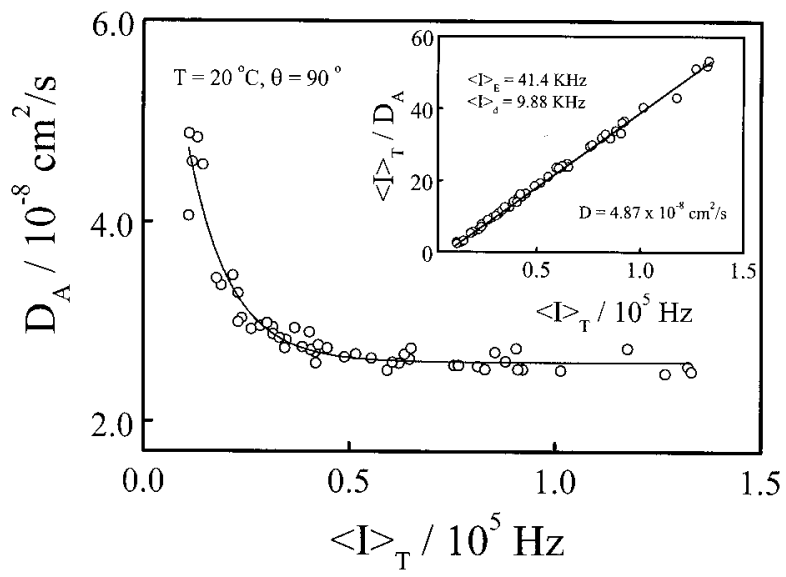

Figure 5. Time-average scattered light intensity $\langle I\rangle_{T}$ dependence of apparent diffusion coefficient $D_{A}$ of the hybrid gel formed at $20^{\circ} \mathrm{C}$, where $\theta=90^{\circ}$ and $\mathrm{D}_{\mathrm{A}}$ was obtained from the initial slope of $S(q, \tau)$. The inset shows a plot on the basis of eq 4 , in which the intercept and the slope lead $\langle I\rangle_{d}$ and $D$, respectively.

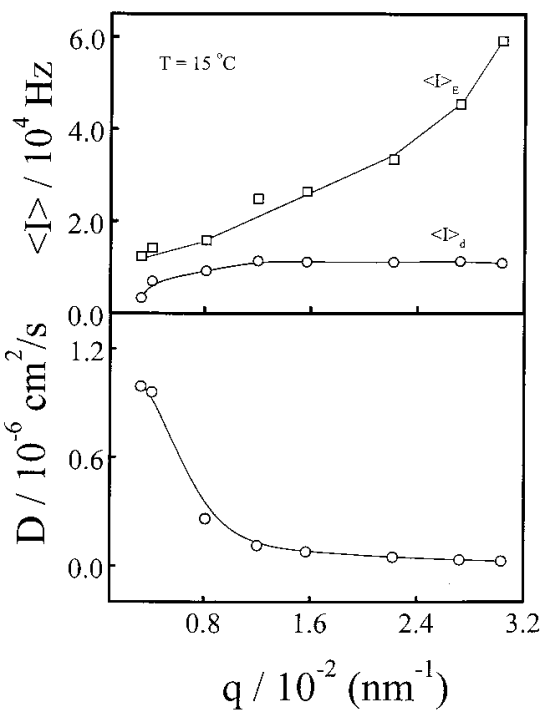

Figure 6. Scattering vector (q) dependence of ensemble average scattered light intensity \langle\rangle$_{E}$, time-average dynamic component of scattered light intensity $\langle I\rangle_{d}$, and collective diffusion coefficient $\mathrm{D}$ of the hybrid gel formed at $15^{\circ} \mathrm{C}$.

magnitude in delay time, namely, $\mathrm{S}(\mathrm{q}, \tau) \sim \tau^{-(1-\mathrm{u})}$, where $\mathrm{u}$ is a critical exponent. In this case, $\mathrm{u} \sim 0.8$, much higher than $\sim 0.4$ reported before, ${ }^{4}$ indicating that the hybrid gel is a resemblance of glasses. ${ }^{25}$

Figure 5 shows that $D_{A}$, calculated from the initial slope of the plot of "In $S(q, \tau)$ vs $\tau$ ", decreases with increasing $\langle I\rangle_{T}$ and approaches a constant when $\langle I\rangle_{T}$ is sufficiently high. The inset is a corresponding plot on the basis of eq 4 . A least-squares fitting of the data leads to $D$ and $\langle I\rangle_{d}$ respectively from the slope and intercept. The relative errors of $D$ and $\langle I\rangle_{d}$ are $\sim 3 \%$ and $\sim 5 \%$, respectively. In this way, we were able to obtain $\left\langle I_{E}\right.$, $\langle I\rangle_{d}$, and $D$ for each given scattering vector $q$. Figure 6 shows that both $\langle I\rangle_{E}$ and $\langle I\rangle_{d}$ increases with $q$. It is known that, at a higher $\mathrm{q}$, the laser light probes a smaller dimension inside the gel. Normally, the scattering intensity decreases as $q$ increases. Here, the increase of $\langle I\rangle_{E}$ with q indicates that the hybrid gel is more homogeneous in a large scale than in a smaller scale. On the other hand, D decreases as q increases. It shows that both $\langle I\rangle_{\mathrm{d}}$ and $\mathrm{D}$ approach constants at $\mathrm{q} \sim$ $1.2 \times 10^{-2} \mathrm{~nm}^{-1}$, suggesting that the correlation length 


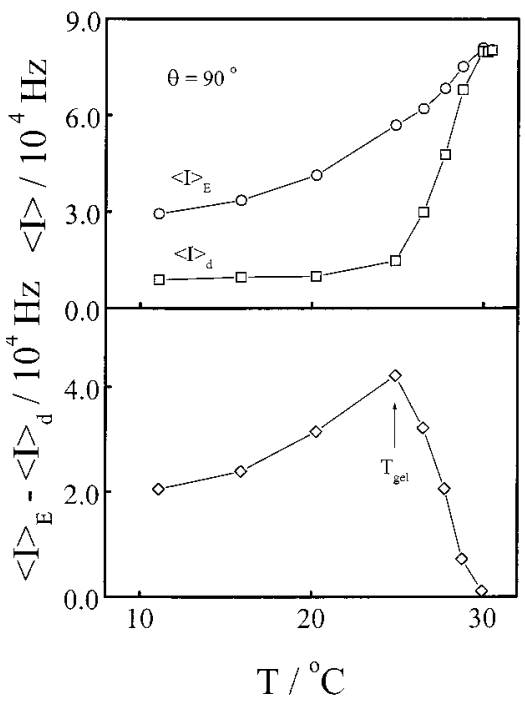

Figure 7. Gelling temperature dependence of ensembleaverage scattered light intensity $\langle I\rangle_{E}$, time-average dynamic component of scattered light intensity $\langle I\rangle_{d}$, and the light intensity difference $(\Delta I)$ between $\langle I\rangle_{E}$ and $\langle I\rangle_{d}$ of the hybrid gel. The arrow shows the gelation temperature $\mathrm{T}_{\text {gel }}$ observed in $\mathrm{a}$ flow test.

of the dynamic fluctuation increases when the light probes a dimension smaller than $80 \mathrm{~nm}$. It is worth noting that the value of $D$ is close to that of the individual microgels in a dilute dispersion.

Figure 7 shows that, for a given $q,\langle I\rangle_{E}$ continuously decreases with the temperature at which the dispersion was aged (i.e., the gelling temperature), but $\langle I\rangle_{d}$ approaches a constant when the gelling temperature is lower than the gelation temperature $\left(\mathrm{T}_{\text {gel }} \sim 25^{\circ} \mathrm{C}\right)$. N ote that $\mathrm{T}_{\text {gel }}$ is a characteristic temperature for a given microgel dispersion, which is different from the gelling temperature. The results in Figure 7 are expected because $\langle I\rangle_{d}$ is only related to dynamic part of the density fluctuation of the hybrid gel. Note that in Figure $1\left\langle R_{h}\right\rangle$ is nearly a constant when $\mathrm{T}<25^{\circ} \mathrm{C}$. Therefore, the gelling temperature affects the gelling rate, the sol/ gel ratio, and the inhomogeneity but not the mobility of those microgels free inside the jam-packed hybrid gel network. The decrease of $\langle I\rangle_{E}$ with the gelling temperature reveals that the hybrid gel formed at a lower gelling temperature is more uniform. In the temperature range $25-30{ }^{\circ} \mathrm{C}$, only some of the microgels are jam-packed. As the gelling temperature decreases, more microgels are attached together so that both $\langle I\rangle_{d}$ and $\langle I\rangle_{E}$ decrease. At $30^{\circ} \mathrm{C}$, all the microgels are free in the dispersion, leading to $\langle I\rangle_{d}=\langle I\rangle_{E}=\langle I\rangle_{T}$. The extrapolations of $\langle I\rangle_{d}$ in the two temperature regions lead to a temperature that is close to the gelation temperature $\left(T_{\text {gel }}\right)$ observed in a macroscopic flow test.

In the past, the high value of $\langle I\rangle_{E}$ was often used to characterize the inhomogeneity. However, our result shows a different picture; namely, the microgel dispersion at $\sim 30^{\circ} \mathrm{C}$ is homogeneous and ergodic; even its $\langle I\rangle_{E}$ or $\langle I\rangle_{T}$ is higher. Therefore, it should be the difference between $\langle I\rangle_{E}$ and $\langle I\rangle_{d}$ that reflects the extent of the inhomogeneity of a given system, not the absolute value of $\langle I\rangle_{E}$ alone. Figure 7 shows that as the gelling temperature decreases, the intensity difference $\left(\langle\mathrm{I}\rangle_{\mathrm{E}}-\langle\mathrm{I}\rangle_{\mathrm{d}}\right)$ reaches a maximum at the gelation temperature $\left(T_{\text {gel }}\right)$. This is because the density fluctuation reaches its peak at $T_{\text {gel }}$. A further decrease of the gelling temperature

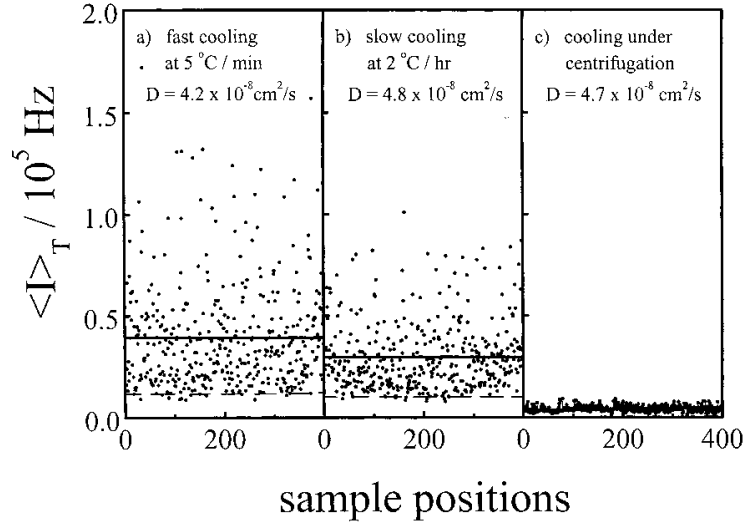

Figure 8. Sample position dependence of time-average scattered light intensity $\langle I\rangle_{\mathrm{T}}$ of the hybrid gel after the microgel dispersion was cooled from $40{ }^{\circ} \mathrm{C}$ to the gelling temperature $\left(15^{\circ} \mathrm{C}\right)$ under different conditions, where the solid and dashed lines represent $\langle I\rangle_{E}$ and $\langle I\rangle_{d}$, respectively.

reduced the difference and resulted in a more uniform hybrid gel.

Since individual swollen microgels are nearly transparent to the laser beam, the inhomogeneity observed in Figure 2 must come from an imperfect packing of the microgels when they were suddenly jammed together. Therefore, if we could slow the cooling process, the swollen microgels would have more time to arrange and pack themselves into a more uniform structure. I deally, if individual collapsed microgels could be closely and neatly stacked together at a high temperature before they were allowed to swell in a slow cool process, we might be able to obtain a uniform hybrid gel. Following this speculation, we designed a series of experiments, in which the microgel dispersion was first kept at 40 ${ }^{\circ} \mathrm{C}$ for $20 \mathrm{~h}$ to ensure an equilibrium and then cool ed to $15^{\circ} \mathrm{C}$ by three different ways: (1) sharply dropped at a rate of $5{ }^{\circ} \mathrm{C} / \mathrm{min}$, (2) slowly decreased at a rate of $2{ }^{\circ} \mathrm{C} /$ $\mathrm{h}$, and (3) centrifuged at $40{ }^{\circ} \mathrm{C}$ for $48 \mathrm{~h}$ before cooled down at a rate of $2{ }^{\circ} \mathrm{C} / \mathrm{h}$ under the centrifugation.

Figure 8 shows that different cooling processes lead to different extents of the inhomogeneity, reflecting in the difference between $\langle I\rangle_{\mathrm{E}}$ and $\langle I\rangle_{\mathrm{d}}$. It is worth noting that Figures $2 \mathrm{c}$ and $8 \mathrm{c}$ are similar, and both of them display an ergodic behavior, i.e., $\langle I\rangle_{E}=\langle I\rangle_{d}$. However, we should note that they represent two completely different physical states; namely, Figure 2c shows the measurement of a uniform and ergodic microgel dispersion in which individual collapsed microgels are under random Brownian motions in water, while Figure $8 c$ was obtained from a hybrid gel, in which individual swollen microgels are neatly stacked together to form a three-dimensional uniform network. Under a white light, such a hybrid gel shows different colors if one views it from different angles. To our knowledge, such a uniform hydrogel has not been obtained before. It clearly demonstrates that the static inhomogeneity is not governed by thermodynamics but dependent on the kinetical gelation process. It is also worth noting that $D$ is nearly a constant, indicating that the gel network less affects the relaxation of individual un-jam-packed microgels. The effects of three different cooling processes on the gel inhomogeneity are schematically shown in Figure 9.

Figure 10 shows that the jam-packing of individual microgels is too fast to be followed in our present setup. This is because the swelling of individual microgels is 


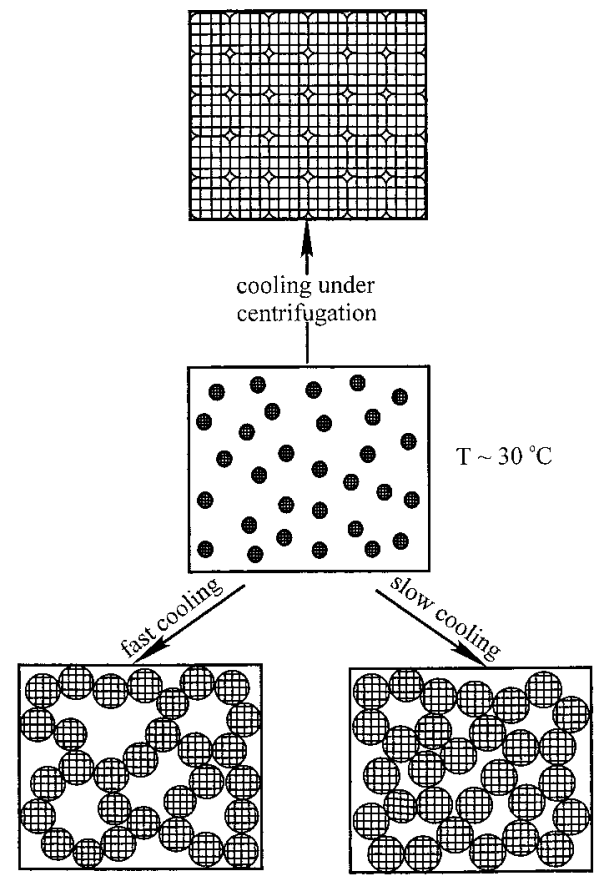

Figure 9. Schematic of influence of different cooling processes on the inhomogeneity of the resultant hybrid gel.

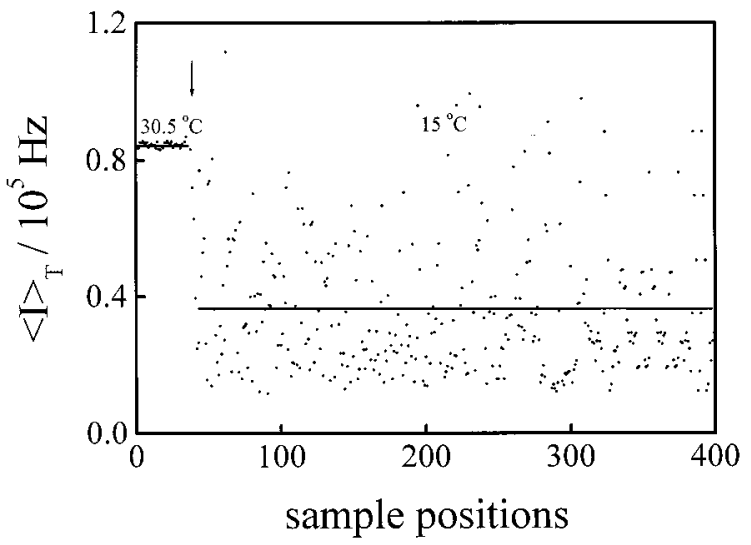

Figure 10. Sample position dependence of time-average scattered light intensity $\langle\mathrm{I}\rangle_{\mathrm{T}}$ of the hybrid gel before and after the temperature jump from 30.5 to $15.0^{\circ} \mathrm{C}$.

much faster than the decrease of the temperature. At $\mathrm{T}=30.5{ }^{\circ} \mathrm{C}$, there is no fluctuation in $\langle\mathrm{I}\rangle_{\mathrm{T}}$, which is expected for a typical dispersion. The second step indicates that the hybrid gel was formed instantly as soon as the dispersion was cooled. Such a formed hybrid gel can undergo a completely reversion back into the microgel dispersion if the temperature is raised to $\sim 30$ ${ }^{\circ} \mathrm{C}$. This is one of the advantages of using such a hybrid gel. It is not difficult to imagine that, before reaching the gelation threshold, individual swollen mi crogels and microgel clusters can be viewed as individual "islands" floating in a solvent "sea", but after the gelation, the jam-packed microgels are interconnected to form a big "continent" (background) and the "sea" changes into many "lakes" (voids). 2,4,26 It was these large and nonuniform voids that led to the speckle pattern of the scattered light intensity. Our results suggest that the static nonergodicity in physical and chemical gels have a similar origin. The only difference is that in chemical gels the clusters are known to be hyperbranched polymer chains and clusters, but in physical gels, they could be formed via a nonuniform interchain association of polymer chains prior to the gelation threshold.

Acknowledgment. The financial support of the CAS Bai Ren Project, the NNSFC projector (29974027), and the HKSAR Earmarked RGC Grants (CUHK/4266/00P, 2160135) is gratefully acknowledged.

\section{References and Notes}

(1) Sato-Matsuo, E.; Orkisz, M.; Sun, S. T.; Li, Y.; Tanaka, T. Macromolecules 1994, 27, 6791 and references therein.

(2) Wu, C.; Zuo, J .; Chu, B. Macromolecules 1989, 22, 633; 1989, 22, 838.

(3) Wu, C.; Chu, B.; Stell, G. Makromol . Chem., Macromol. Symp. $1989,45,75$.

(4) Norisuye, T.; Inoue, M.; Shibayama, M.; Tamaki, R.; Chujo, Y. Macromolecules 2000, 33, 900 and the references therein.

(5) Brown, W. Dynamic Light Scattering, the Methods and Applications; Clarendon Press: Oxford, 1993.

(6) Bastide, J .; Leibler, L. Macromolecules 1988, 21, 2647.

(7) Panyukov, S.; Rabin, Y. Macromol ecules 1996, 29, 7960; Phys. Rep. 1996, 269, 1.

(8) Hecht, A. M.; Duplessix, R.; Geissler, E. Macromolecules 1985, 18, 2167.

(9) Mallam, S.; Horkay, F.; Hecht, A. M.; Geissler, E. Macromolecules 1989, 22, 3356.

(10) Horkay, F.; Hecht, A. M.; Mallam, S.; Geissler, E.; Rennie, A. R. Macromolecules 1991, 24, 2896.

(11) Cohen, Y.; Ramon, O.; Kopelman, I J .; Mizrahi, S. J . Polym. Sci., Polym. Phys. Ed. 1992, 30, 1055.

(12) Shibayama, M.; Tanaka, T.; Han, C. C.J . Chem. Phys. 1992 97, 6829.

(13) Rouf, C.; Bastide, J .; Pujol, J . M.; Schosseler, F.; Munch, J . P. Phys. Rev. Lett. 1994, 73, 830.

(14) Pusey, P. N.; Van Megen, W. Physica A 1989, 157, 705.

(15) J oosten, J . G. H.; Gelade, E.; Pusey, P. N. Phys. Rev. A 1990, $42,2161$.

(16) J oosten, J . G. H.; Mccarthy, J . L.; Pusey, P. N. Macromolecules 1991, 24, 6690 .

(17) Fang, L.; Brown, W. Macromolecules 1992, 25, 6897.

(18) Tanaka, T.; Hocker, L. O.; Benedek, G. B. J . Chem. Phys. $1973,59,5151$

(19) Horkay, F.; et al. Macromolecules 1993, 26, 3375

(20) Pelton, R. H.; Chibante, P. Colloids Surf. 1986, 20, 247.

(21) Wu, C.; Zhou, S. J . Polym. Sci., Polym. Phys. Ed. 1996, 34 1597.

(22) Chu, B. Laser Light Scattering, 2nd ed; Academic Press: New York, 1991.

(23) Berne, B.; Pecora, R. Dynamic Light Scattering; Plenum Press: New York, 1976

(24) Wu, C.; Zhou, S. Q. Macromolecules 1995, 28, 8381.

(25) Martin, J . E.; Wilcoxon, J .; Odinek, J . Phys. Rev. A 1991, 43, 858.

(26) Norisuye, T.; Shibayama, M.; Nomura, S. Polymer 1998, 39, 2769.

MA010672P 